Dr. Beverzy R. TuCKer, Richmond, Va.: I am of the opinion that we have not laid enough stress on the hereditary factor in mental diseases. The doctor spoke of inherited aptitudes. I had occasion at one time to investigate the manufacture of artificial eyes, and it was found that it could not be very successfully carried out in this country without the importation of Bohemians trained for generation after generation in this particular work. I believe that it is an inherited aptitude, and that the simpler aptitudes involving the motor factors are the easiest to carry, and then we come to the ones below. I do not believe that people "go" insane. $I$ believe that when physical disease is induced, for instance by toxemia, as by alcohol, this releases inhibition and then we see the individual stripped of all his veneer, and the man who becomes maniacal under alcohol has a maniacal tendency. We do not lay enough stress on the inheritance factor. I am of the belief that neither poison nor anything else can make a person insane unless he has an inherited tendency. In other words, a normal mental mechanism remains a normal mental mechanism, and the only influence that disease has on it is to cloud it. Any well-formed syndrome that is manifest has aiways been present. A person does not "become" insane; he las always been insane, and some particular determining factor makes that apparent.

Dr. Lawrence B. Pilsbury, Lincoln, Neb.: I purposely avoided going into personality very much; personality is too big a problem. We do not know what personality is, we simply know some of its manifestations. As to somatic and psychogenic causes, they are very hard to separate. It is as hard to separate the potentially somatic cause from the potentially psychogenic cause as it is to bridge the gap between the body and the mind. Dr. Sterne speaks of fear. We do not know what fear is, but we know some of the manifestations of fear. Probably some of the ductless glands have something to do with fear. Dr. William James, in elaborating his theory, points out the fact that we are, so to speak, afraid because we run; that we do not run away from an object, a bear for example, because we are afraid. We first begin to run-we first react-and then afterward we realize that we are afraid. Of course, there is simply an idea or an emotion with a physical expression or manifestation, but we cannot have physical reaction without a cause, and the cause in this instance is a stimulus, probably an optical stimulus-we see a bear and begin to run, and we are afraid. The whole thing has a material and physiologic basis, and how to separate the emotion of fear from its manifestations I do not know.

Dangerous and Harmless Color Blindness. - In a recent investigation made by the United States Public Health Service, an important distinction was made between persons who are only slightly color blind and those who are dangerously color blind, that is, unable to distinguish at all times between red and green. The results of the investigation form a working basis on which examiners may discover members of the latter class and exclude them from all government positions in which the reading of colored signals is a part of the work. It was, found by various tests that the latter class includes persons who are able to see three or fewer colors in the spectrum, those who see more than three colors but have the red end of the spectrum so shortened as to prevent the recog. niton of a red light at a distance of 2 miles, and those with a central scotoma for green and red. In making the tests, the health service officials used the Edridge-Green color lantern in preference to colored yarns. In the course of the tests, 1,000 persons were examined by means of the lantern, in order to determine both its value and the effect of refractive conditions and lesions of the eye on color perception. The examinations also revealed the fact that color blindness occurs in about 8.6 per cent. of men and 2.2 per cent. of women, if we exclude those who are able to distinguish five colors in the spectrum. Among workers in occupations requiring the ability to recognize colored signal lights, dangerous color blindness prevails to the extent of 3.1 per cent. among men and 0.7 per cent. among women. It appears the most frequently in eyes affected with mixed astigmatism, and the least frequently in those that show no refractive error.

\section{SOME VITAL PHASES OF FRACTURES OF THE JAWS *}

\author{
CHALMERS J. LYONS, D.D.Sc. \\ ANN ARBOR, MICH.
}

The subject of fractures of the jaws is one that has attracted the attention of medicine since the days of Hippocrates. It also is a subject that was of considerable interest to dentistry. long before it became a profession. Indeed, many of the principles laid down by Hippocrates can be used today with a moderate degree of success. It was Hippocrates who first originated interdental ligation. It was also Hippocrates who condemned the use of bandages alone as a procedure in the treatment of fractures of the jaws.

In presenting the subject of fractures of the jaws at this time, we shall touch on only two or three vital phases of the subject. The first phase which we shall attempt to discuss will be the process of repair. We use the word "discuss" advisedly, because in our present state of knowledge of the subject, we can only discuss it. We are not in a position to make any dogmatic statements as to just what takes place in the process of repair of bone.

Just what takes place in the behavior of the tissues in the process of repair of bone is a question offering a diversity of opinion. There seems to be an antagonistic state of opinion about the theory of bone? growth. There are two schools that are contending for the maintenance of their theories along this line: One contends that the periosteum is a limiting membrane without the property of osteogenesis; the other maintains that the periosteum is an osteogenetic membrane and can go on functionating, developing and nourishing new bone.

Havers, in 1692, gave the first accurate account of osseous structure and described the periosteum as simply a connective tissue, limiting and vascularizing membrane.

Antoine de Heyde, in 1684, made some observations on frogs and determined that callus was formed by calcification of a blood clot extravasated around broken bone ends.

In the middle of the eighteenth century, Duharnel brought out the generally accepted theory of the function of the periosteum. His view was that the periosteum became thickened and succulent around a fracture and by pushing the new tissue in among the fragments it formed a callus. In his experiments, he discovered a layer of cells lying next to the bone. 'To this layer, he gave the name cambium layer. This layer of cells between the true periosteum and the bone is recognized in the bone work now being done in Europe.

Macewen ${ }^{1}$ is emphatic in the statements that the function of the periosteum is simply a limiting membrane and does not have the property of osteogenesis. He made many experiments on dogs. In these he seems to prove that the periosteum does not produce new bone. In many instances, he reports that the whole radius was removed, leaving the periosteum. He finds in these cases that after several weeks no new bone is formed. In his experiments, he finds no place where the periosteal flaps produced new bone. He also finds that where the periosteum was

* Read before the Section on Stomatology at the Sixty-Ninth Annual Session of the American Medical Association, Chicago, June, 1918 .

1. Macewen, Sir William: The Growth of Bone, New York, Tha Macmillan Company, 1912. 
not intact, there was a marked hypertrophy of the bone, showing that periosteum limits bone growth.

These experiments of Macewen are substantiated by similar experiments by Cohn and Mann. Their experiments included transplanting of bone denuded of periosteum into muscle, into the medullary canal and into newly made bone defects. Some of these transplants were covered with periosteum and others were not. It was found that isolated bone grafts did not act as foreign bodies and were not absorbed after sixty days, but showed a tendency to outgrowth. They also made experiments in which the periosteum per se was transplanted around the carotid artery. This did not show bone proliferation. They came to the conclusion that the periosteum was not at all essential to the healing of a fracture.

Other investigators in this country have made similar experiments with similar results. These investigations, with others, represent the work of the contenders of the theory that the periosteum is simply a limiting membrane. The other class of investigators in bone work contend that these experiments must have been carried out with simply the outer layer of the periosteum, which is a limiting membrane, and that the inner layer, or the cambium layer, the layer which Hey Groves terms the epiostial layer, or the osteogenetic layer of the periosteum, was not considered.

Our experiments at the University of Michigan hospitals do not accord with the theory of Macewen. We have found that periosteum transplanted into the muscle tissue will produce new bone. This new growth seems to have all the properties of true bone; its blood supply, its bone cells and the process of true osteogenesis seem to be normal.

This deposition of bone differs from other calcific deposits which may take place in the body, such as in the spleen, liver, kidney, etc., under certain pathologic conditions, so that we feel quite positive that under suitable conditions, true bone may form from the periosteum.

\section{Hey Groves summarizes as follows:}

The periosteum is chiefly a limiting membrane of the bone. The dense bone can live, grow, undergo repair, and produce fresh periosteum after the latter has been removed. In young bones it is possible to remove the periosteum in such a way as to produce an osteogenetic membrane, this being probably due to the lifting up of the epiosteum with the periosteum. In adult bones this is impossible except after trauma or an inflammation.

In the repair of fractures of the jaws, we feel that the retention of the periosteum is highly desirable, because its removal takes away much of the epiosteum, and, also, because it affords a ready means of vascularization.

While there is a wide diversity of opinion regarding the function of the periosteum in bone repair, all investigators seem to agree as to the function of the compact and cancellous bone in the process of repair.

Ollier proved that quite apart from the periosteum and the nuarrow, compact bone could live and produce new bone and undergo the callus repair of fracture. The deep surfaces of bone, like the superficial surfaces, are capable of osteogenesis under suitable stimulus.

Axhausen has shown that the wide haversian canals contain active osteoblasts and favorable conditions for new bone formation. It has been observed in gunshot fractures in France that when the ends of the frag- ments have been exposed and laid bare, by stimulating the ends by a drill, in a short time buttons of granulation tissue would be pushed out and would soon cover the entire ends of the bones. These ends could then be put in apposition, and union of fragments would result. In our clinics, we have had to resort to this method in some old ununited fractures, in which we have had the process of repair again started. It is our opinion that compact bone, if it has the proper blood supply, is quite independent of either endosteum or periosteum for bone growth and for bone repair.

The whole process of repair of bone is fundamentally that which takes place in the union of the soft parts. In fractures of the bone we may have primary union, or, we may have secondary union by granulation tissue; this granulation tissue differs from granulation tissue of the soft parts in that it is osseous in character. The injured tissues, infiltrated with blood, soon become invaded by leukocytes and effused blood plasma. Firbrinous coagulation takes place and the ends of the fragments are embedded in a dense, ill defined mass of firm, cellular exudate. The periosteum becomes much thicker, softer and more vascular; a thin layer of gelatinous or viscid liquid is found between it and the bone for a short distance from the edge of the fracture. In about fourteen days, the effused blood is completely absorbed, leaving a firm, dense, cellular, vascularized, partly organized mass of granulation tissue. The bone then undergoes rarefying osteitis, and the fracture becomes fixed. This is known as the "provisional callus."

While this process is going on, similar changes are taking place in the cancellous bone, and the "internal callus" is formed in the same manner. Ossification then takes place, thus completing the process of repair. While the callus is forming, the process of repair is going on in the contiguous soft parts, and they regain their normal condition and function.

Very briefly, the foregoing is what seems to take place in the process of repair of fracture of the jaws. Occasionally there may be an excess of rarefying osteitis, and a lack of production of osteoblasts, so that the callus may not ossify. In these cases, the bone is absorbed for a considerable distance between the ends of the fragments, and we have established a false joint or pseudo-arthrosis.

When an open or compound fracture becomes infected, suppuration ensues, and the process of repair is slower, because the suppuration of the wound delays or prevents the formation of the provisional callus, and it has to depend on the formation of the internal callus, which is not so favorable for rapid repair. In these cases, the callus is larger and more irregular than that which we see after simple fractures, when the process of repair takes its normal course.

Another phase of this subject which we shall consider briefly is some of the complications which occur in fracture of the jaws. Fractures of the jaws will differ from fractures in other parts of the body in that they are more liable to infection on account of the close proximity to the bacteria-laden fluids of the oral cavity. We rarely find infection present in simple fractures of the jaws, but it is quite common in compound fractures.

A factor which must be considered in relation to infection in fractures of the jaws is the presence of alveolar abscesses, which may be existing at the time of the fracture or may be superinduced by the injury. These will greatly delay the process of repair and 
should be eradicated before repair can be expected to take place.

In fracture of the maxilla, the antrum is frequently involved and may become infected. This makes another serious complication. It means clearing up the infection in the antrum before the process of repair will go on in a normal way.

Another rare complication in fracture of the maxilla is the fracture of the brain case, in which coma or even death may result immediately. Secondary hemorrhage is one of the complicating problems that is quite common in fractures of the jaws on the battle fields of Europe. This comes on after suppuration has been established and is the result of infective inflammation causing a disintegration of the hemostatic thrombosis, or ulceration or sloughing of the valls of the vessels. This secondary hemorrhage may occur any time between the beginning of the process of repair and the complete repair of the fractured blood vessels.

The laceration or severing of sensitive nerve trunks will lead to anesthesia of all of the parts peripheral to the fracture, and neuralgia is a common sequela.

Trismus is an early local complication that is nearly always present. This is usually brought on by the violence that is produced on the soft tissues and the temporomandibular articulation. In extreme cases, where it is not possible to make the reduction at once, the trismus may be so pronounced that a general anesthetic may have to be resorted to in order to obtain sufficient relaxation to make a diagnosis and subsequent reduction.

In gunshot fractures, there are other local complications, such as extensive laceration of the soft parts, which will greatly add to the difficulty of making a diagnosis and prosecuting the treatment. In these cases, there will usually be greater splintering and fissuring of the bone than in the fractures in civil practice. It is reported that in every case of gunshot fracture of the jaws, infection of the wound ensues immediately, which greatly interferes with the process of repair.

In the work at the front on fracture of the jaws, the history shows that many of these cases are not treated until some time after the injury. Here a new complication arises. In these cases, frequently large masses of cicatricial tissue have formed, and a marked deformity is present. Under these conditions, a clear and definite diagnosis may be difficult, and the successful reduction of the fracture made arduous.

Another local complication, which is almost universal in gunshot fractures of the jaw and sometimes met with in civil practice, is loss of substance of the bone. Here again difficulties arise in making a successful reduction and subsequently retaining the fragments in normal relation.

Under the general complications of fractures of the jaws, there are a few factors that must not be overlooked. There are certain diseases which, when present, seem to hinder the process of repair. Such discases as syphilis, alcoholism, tuberculosis and such chronic diseases as will cause a marked lowering of the vitality of the patient. These conditions will always delay union and may prevent it entirely.

It will not be out of place, perhaps, in this brief paper to say something regarding the prognosis in fractures of the jaw. The prognosis must vary greatly according to the location of the fracture, the character of it, the complications which are present or which fcllow and the age and resistance of the patient. The time which elapsed between the injury and the reduction of the fracture will also influence the prognosis. The prognosis should take into account several points: First, the effect of injury in respect to a favorable or unfavorable termination of the case; second, its simple or complicated course; third, the influence of each complication; fourth, the time required for recovery; and, fifth, the result as to normal occlusion of the teeth and normal functions of the jaws. The younger the patient, the more favorable the prognosis, because in the young fractures unite more easily and promptly than in the adult. If the fracture has existed two or three weeks previous to its reduction, and if there is considerable movement of the fragments, consolidation will not take place as rapidly after the parts have been brought into normal relation as it would if reduction were made at the time of the injury.

The presence or absence of infection plays a very important rôle in the duration of the treatment. In a simple fracture of the mandible where the fragments remain or are replaced in perfect contact, repair will take place at once without any untoward symptoms, without deformity or malocclusion, and without detriment to the functions of the jaws. If the fragments are not quite in apposition in simple fracture, but nearly so, the prognosis will be almost as favorable, although it will take somewhat longer for repair to take place and there may be some slight deformity manifested in malocclusion of the teeth.

All compound, comminuted and complicated fractures, which in their very nature present additional obstacles in the way of complete reduction, may not present so favorable a prognosis. In fractures of the superior maxilla, the fragments will not be subjected to muscle strain as those in the lower jaw, and the retention of the fragments in normal position will not be so complicated. Fractures of the condyle with displacement offer less in the way of favorable prognosis than any other fracture of the lower jaw. In these injuries there usually will be produced a traumatism in the temporomandibular joint which may later result in ankylosis.

Gunshot fractures of the jaws, which are necessarily in most cases compound and comminuted, are in a much less degree amenable to treatment than most other fractures. Splints for supporting the fragments must necessarily be more complicated, and infection is always present. All of these factors must be taken into consideration by the operator in forming a judicious prognosis in the treatment of fractures of the jaws.

Time will not permit us to discuss at length the treatment of fractures of the jaws. The treatment of these fractures consists in the fulfilment of three principal indications:

1. Reduce the broken fragments.

2. Retain the parts in normal relation until consolidation has taken place.

3. Prevent or control inflammatory processes.

No hard and fast lines can be drawn relative to the treatment of fractures of the jaws, for each case will present an entirely different problem.

In the treatment, the operator should aim to establish normal forms and normal function. The different forces, such as muscular tension, the force of gravity, movements of the tongue, etc., which tend to displace the fragments, must be taken into consideration in determining the methods of treatment. 
Individual ingenuity must ever play an important rôle in the treatment of fractures of the jaws. Equally successful results will be obtained by means and methods that are wholly different. In the construction of splints, the aim should be so to design them that all parts of the mouth may be kept clean, without which normal repair will not take place.

\section{ABSTRACT OF DISCUSSION}

Dr. Thomas L. Gilmer, Chicago: In war, especially in trench warfare, injuries above the clavicle occur in the majority of cases; therefore, the jaws are involved often. It is almost impossible to have a fracture of the mandible that is not compound. And another complication follows that oneinfection. It is frequently seen in cases of fracture in the mouth. It does not delay or prevent union, provided the infection is very slight. If the infection is extensive, make an external opening and drain to prevent destruction of tissues.

$I$ have not seen a single case of traumatism of the temporomandibular joint as a result of fracture in civil practice. In the treatment of these cases the main object is to approximate the fragments. The fracture will unite more quickly if the fragments are not immobilized. There seems to be a greater osteogenetic influence where there is slight movement. I have employed an orthodontist extension arch, fastening it to the teeth on the upper jaw as well as to the lower, and then securing the upper bar to the lower bar by wires. The mouth can be opened every day. If you do not use immobilizing appliances, the patient must be instructed that under no circumstances is he allowed to use food which will require mastication.

Dr. Truman W. Brophy, Chicago: Some years ago I saw in Europe a method of passing a bar on one side which had several wire bands adjusted to the teeth which ran on a slide fixed to the other jaw and also were fixed by bands to the teeth. These bars run in slides, thereby holding the long fragment where it belongs and thereby preserving the occlusion of the teeth. We must never forget that we can hold the teeth in occlusion on the side of the long fragment. In case a great deal of bone has been lost, cicatrization will preserve the lines of the face. When a portion of bone has been lost, there is no better treatment than by following the principles laid down by Dr. Gilmer. If only the third molar is left, let it hold the fragment in its proper position. The teeth on that side will be held in position and the intervening space will be filled in by new bone arising from the periosteum. If we can hold these in approximation very often much bone will form. By employing the method of wiring the tecth together we succeed in getting a good union, though part of it is cartilaginous, or rather fibrous. The proper occlusion of the teeth is preserved.

Dr. Arthur D. Black, Chicago: It might be of interest to know that in the Plastic and Oral Surgery Course of the Army the men are instructed that in every place where there is a fracture they are to make an external incision into the open space between the ends of the bone.

Dr. Jonn E. Nyman, Chicago: In treating these fractures we need some means of mechanical fixation quite apart from that supplied by the teeth. In one case of multiple fracture we used platinum screws and wires because we thought that they could be sterilized more easily. The result was excellent.

Dr. Arthur Zentler, New York: Dr. Depage and his associates use a new nomenclature for jaw fractures, terming them according to location, premolar, retromolar, retropremolar, and right or left. At LaPanne they have adopted a new method of treatment. For instance, in a case of retromolar fracture of the mandible, they attach a band to a tooth in the part which is not loose. Another band is attached to a tooth in the loose portion. To these bands a heavy expansion arch wire was previously soldered which extends and overlaps a similar wire soldered on the other band. The fracture is reduced with the patient anesthetized, and with soft solder the overlapping ends of the wires are united in the mouth.
Dr. A. W. McCullougr, Pittsburgh: The first essential in reducing fractures is to secure apposition. Dr. Gilmer's wiring becomes rather tiresome to the patient after a few days and he wants it removed. I have used Angle's bands on the teeth, together with rubber bands attached to the upper and lower Angle's on teeth. Sometimes it works very nicely and gives the patient an easier condition of the muscles than we get when the jaws are wired together with immobilization. Regarding bands, we have used three or more bands, with bar attachments; bands of the Lukens design, that may be adjusted easily or changed, and which I prefer to cementing.

Dr. Frederick B. Moorehead, Chicago: Dr. Lyons' experimental work does not tally with the principles of bone regeneration as we understand them. It is understood that regeneration of bone must come from the osteoblasts, and that the periosteum per se has no capacity of bone regeneration. Dr. D. B. Phemister of Chicago has proved that bone transplants without periosteum are quite as vital in their function as those with the periosteum. One may take a section of bone from which the periosteum has been removed and then remove the subperiosteal layer by cutting it away and it will be found that that piece of bone has a certain capacity for regeneration, which must, of course, be found in the osteoblast. It must be true that in transplanting periosteum into muscles, as reported by Dr. Lyons, some of the osteoblasts were retained in the periosteum in its removal from the bone. The French method of treating fractures of the jaw is not to immobilize. Where there is no displacement and no tendency to displacement in muscle movement, a fracture of the jaw is more satisfactorily treated without immobilization than with it. Fixation and immobilization are necessary only where there is displacement, which cannot be controlled except by immobilization. It is doubtless true that the amount of movement occasioned by the muscle only acts as a stimulant and hastens repair.

Dr. Chalmers J. Lyons, Ann Arbor, Mich.: I think we are getting away from fixation of the jaw. We must retain the fragments when there is displacement. In mandibular fractures, where there is little or no displacement, we are getting away from the intradental ligation. The experience in fracture work of the men at the front is going to bring out many new things. Ordinarily, where there is infection of the mouth we can get along much better without fixation. In gunshot fractures I question whether intradental ligation has much of a place on account of infection. The point Dr. Gilmer brought up about external openings is a method we have resorted to in many cases. If an abscess forms you must drain. If pus forms around a fragment, repair is going to be retarded. It is much better to make an external opening at once. Relative to Dr. Gilmer's point of the osteoblasts lyincr just under the periosteum, we all recognize this fact, and it is to this layer that Hey-Groves has given the name of epiosteum. It is the osteogenetic portion of the periosteum. Without this layer of cells, the periosteum has not the property of producing new bone.

Improved Approach to Kidney.-Dr. G. Kolischer descriles a new incision which he had devised for exposing the kidney (Interstate Mcdical Journal, 1918, 25, 128) and which reduces the amount of destruction to the soft tissues of the abdominal wall. The patient lies on the operating table in such a way as to make his flank protrude. The skin incision is made half way between the twelfth rib and the crista ilii, and parallel to the rib, beginning at the anterior edge of the latissimus dorsi, and slanting downward the center of an imaginary line running from the umbilicus toward the spina superior ilii. The incision extends down through the fascia. Both lips of the wound are undermined for 2 or 3 inches, Retract the flaps so as to expose the external oblique. Separate the fibers of this muscle bluntly in the center, and also those of the internal oblique, including the transversalis. The fascia transversalis is split vertically with the knife and spread wide open with retractors so as to give a free exposure of the kidney. The wound is closed by reuniting the fasciae and approximating the skin edges. Tension sutures are also inserted. 